\title{
Predição da Restrição do Crescimento Fetal pela Biometria do Diâmetro Transverso do Cerebelo
}

Prediction of Fetal Growth Restriction by Biometry of the Transverse Diameter of the Cerebellum

Luiz Nery ${ }^{1}$, Antonio Fernandes Moron², Luiz Kulay Junior ${ }^{2}$

\section{RESUMO}

Objetivo: avaliar, em gestações de risco, a acurácia da medida do diâmetro transverso do cerebelo (DTC) e da relação diâmetro transverso do cerebelo/circunferência abdominal (DTC/ $C A)$ para detecção de restrição de crescimento fetal (RCF).

Método: foi realizado um estudo prospectivo transversal envolvendo 260 pacientes com idade gestacional entre a $28^{a}$ e a $40^{a}$ semana. Os fetos foram avaliados por meio da ultra-sonografia, obtendo-se o DTC e CA. Fetos com DTC menor que o percentil 10 para idade gestacional ou com relação DTC/CA acima do percentil 90 (>14,6) foram classificados como suspeitos para RCF. Após o parto, avaliou-se a acurácia da medida do DTC e da relação DTC/CA para a predição da RCF, utilizando-se como critério diagnóstico o peso do recém-nascido menor que o percentil 10 para idade gestacional.

Resultados: após o parto, foram identificados 79 recém-nascidos com $R C F(30,4 \%)$. O DTC foi adequado para a idade gestacional em 74 desses fetos (93,7\%), e compativel com pequeno para a idade gestacional em apenas 5 (6,3\%). A sensibilidade, especificidade, valor preditivo positivo, valor preditivo negativo e acurácia do DTC na predição de RCF foi de 6,3, 93,4, 29,4, 69,5 e 67\%, respectivamente. A relação DTC/CA maior que 14,6 identificou 59 dos 79 fetos com RCF, com 27 falso-positivos e 20 falso-negativos, apresentando, portanto, sensibilidade de $74,5 \%$, especificidade de $85,1 \%$, valor preditivo positivo de 68,6\%, valor preditivo negativo de $88,5 \%$ e acurácia de $81,9 \%$.

Conclusões: a medida isolada do DTC não é bom parâmetro para rastrear RCF, porém a relação DTC/CA acima do percentil 90 mostrou-se eficaz para detectar fetos com restrição de crescimento.

PALAVRAS-CHAVE: Biometria fetal. Cerebelo. Restrição do crescimento fetal.

\section{Introdução}

A restrição do crescimento fetal (RCF), anteriormente denominada retardo de crescimento intra-uterino, acomete em média 2 a $10 \%^{1,2}$ de to-

\footnotetext{
${ }^{1}$ Disciplina de Ginecologia e Obstetrícia da Universidade Estadual de Maringá

${ }^{2}$ Departamento de Obstetrícia da Universidade Federal de São Paulo/Escola Paulista de Medicina

Correspondência:

Luiz Nery

Rua Saint Hilaire 244 - Zona 05

87015-160 - Maringá - PR

Tel: (44) 224-9098 - Fax (44) 224-5522

e-mail: nery@wnet.com.br
}

dos recém-nascidos (RN) e ainda é considerada situação de extrema complexidade. Apesar dos notáveis avanços da medicina nas áreas de obstetrícia, ultra-sonografia e neonatologia, os RN com RCF continuam apresentando elevada morbimortalidade perinatal ${ }^{3}$. Classicamente, considera-se que um feto apresenta RCF quando o seu peso estimado situase abaixo do percentil 10 para a idade gestacional ${ }^{4}$. O diagnóstico antenatal da RCF depende, portanto, obrigatoriamente da estimativa correta da idade gestacional (IG), o que nem sempre é tarefa fácil. Estima-se que 20 a $40 \%$ das pacientes desconhecem a data da última menstruação (DUM), e aproximadamente $15 \%$ informam erroneamen- 
te o último período menstrual ${ }^{5}$. Soma-se a isso o fato de que muitas gestantes têm sua primeira avaliação pela ultra-sonografia (USG) tardiamente, quando a estimativa da IG apresenta erro intrinseco grande e as medidas fetais podem estar reduzidas em decorrência do processo de restrição do crescimento fetal, o que pode induzir o ultrasonografista a subestimar a IG real. Portanto, mesmo para obstetras e ultra-sonografistas experientes, o diagnóstico antenatal de RCF ainda constitui um desafio.

Desde 1984 a literatura ${ }^{1-3,6,7}$ tem apontado a medida do diâmetro transverso do cerebelo (DTC) como bom parâmetro para determinação da IG, inclusive nos exames de segundo e terceiro trimestres e em fetos com RCF. Reece et al. ${ }^{1}$ observaram que, ao contrário de outros parâmetros biométricos como o diâmetro biparietal (DBP), fêmur e circunferência abdominal (CA), a biometria cerebelar não era afetada em fetos com restrição de crescimento. Portanto, nesses fetos o DTC seria elemento importante na determinação correta da IG. Apesar de a maioria dos trabalhos apontarem que o DTC não se alteraria nos fetos com $\mathrm{RCF}, 2,3,8-12$, alguns indicam que as medidas do cerebelo poderiam estar reduzidas nesses fe$\operatorname{tos}^{13,14}$.

Diante da redução de oferta de oxigênio e nutrientes, o feto tenta compensar a situação por meio da redistribuição da sua circulação. Essa adaptação, conhecida como "centralização fetal" consiste na vasodilatação seletiva de alguns territórios (coração, cérebro e supra-renais) e vasoconstrição de todos os outros ${ }^{3}$, o que resultaria em um crescimento abdominal menor do que o esperado para a IG. Baseado nesse crescimento desproporcional, surgiram alguns parâmetros ultra-sonográficos para auxiliar no diagnóstico de RCF, como a relação circunferência cefálica-circunferência abdominal, a relação fêmur-abdome ${ }^{4}$, e mais recentemente, a relação diâmetro transverso do cerebelo-circunferência abdominal (DTCCA) ${ }^{1-3,7-12}$.

Ao contrário da CA, o cerebelo, segundo a maioria dos autores, parece não se alterar mesmo em casos de RCF grave. Portanto, a relação cerebelo/abdome fetal (DTC/CA) elevada (acima do percentil 90) seria uma boa forma de rastrear casos de RCF assimétrico. O valor exato da relação DTC/CA a partir do qual se suspeitaria de RCF varia conforme as curvas de DTC e CA utilizadas pelos diversos autores. A maioria aponta valores situados ao redor de $15,7( \pm 1,7)^{9}$ até $16,7( \pm 1,8)^{10}$.

Motivados pelos achados ainda conflitantes da literatura e pela inexistência de dados nacionais a esses respeito decidimos avaliar em nossa população a eficácia da medida isolada do DTC e da relação DTC/CA no rastreamento de RCF em pacientes de risco.

\section{Pacientes e Método}

Realizou-se estudo prospectivo do tipo transversal, entre janeiro de 2000 e agosto de 2001, para o qual foram selecionadas inicialmente 420 pacientes de alto risco para RCF. Foram incluídas pacientes com hipertensão arterial crônica (HAC), doença hipertensiva específica da gestação (DHEG), diabete melito com vasculopatia, cardiopatas, grávidas com infecções congênitas, oligoidramnia, colagenoses, desnutrição, anemias, tabagistas e usuárias de drogas durante o período gestacional. As pacientes eram oriundas do Ambulatório de Pré-natal de Alto Risco do Hospital Universitário de Maringá (HUM) e da Rede Pública Municipal.

Foram incluídas pacientes com gestação única e IG igual ou superior a 28 semanas. Todas eram eumenorréicas, conheciam a DUM e tinham USG antes da $20^{\mathrm{a}}$ semana concordante com a IG menstrual. Foram excluídas as pacientes que, durante o estudo, tiveram óbito fetal; aquelas cujos fetos apresentavam malformação congênita e as que tiveram parto fora da instituição onde realizou-se o estudo. O estudo foi aprovado pela Comissão de Normas Éticas e Regulamentares da Universidade Federal de São Paulo (UNIFESP) e as pacientes assinaram o termo de consentimento informado.

As 420 gestantes que preencheram os critérios de inclusão foram avaliadas por meio da USG. Porém, $160(38,1 \%)$ foram excluídas, por diversos motivos: $75(17,9 \%)$ por perda de seguimento, 70 $(16,7 \%)$ por visualização inadequada do cerebelo e $15(3,5 \%)$ por óbito fetal. Permaneceram no estudo 260 pacientes $(61,9 \%$ da amostra inicial). A idade dessas pacientes variou de 15 a 42 anos (média 28 anos) e a paridade, de 1 a 6 gestações (média de 3 gestações). Quanto aos fatores de risco para $\mathrm{RCF}$, houve predomínio das sindromes hipertensivas $(18,1 \%$ com DHEG, $8,1 \%$ com HAC), seguidas de tabagismo $(11,5 \%)$ e doenças infecciosas $(6,2 \%$ com citomegalovirus)

Para o cálculo da amostra considerou-se incidência média de $7 \%^{1,2}$ de RCF na população geral. A partir desses dados chegou-se a uma amostragem de 204 fetos, com erro alfa de 5\%, erro beta de $5 \%$ e poder de $95 \%$. O tamanho amostral foi calculado a partir do programa EpiInfo 6. 
A primeira parte do exame ultra-sonográfico foi realizada por dois observadores que avaliaram o DBP, circunferência craniana (CC), CA e crescimento fetal, liquido amniótico e placenta. A mensuração do DCT foi realizada somente pelo primeiro autor (LN), utilizando aparelho de ultrasonografia da marca GE Logiq 500, Pro-series, de varredura convexa eletrônica, com freqüência de emissão de 3,5 MHz. O cerebelo era identificado na fossa posterior, em plano paralelo aos ventrículos laterais, tendo como pontos de referência: tálamo, cavum do septo pelúcido e terceiro ventrículo ${ }^{4}$. Girando-se o transdutor com inclinação de 30 graus, obteve-se a visualização ideal do cerebelo. Em seguida procedeu-se à medida do DTC em milímetros, de uma margem externa à outra dos hemisférios cerebelares ${ }^{7}$. Cada paciente submeteu-se a apenas uma USG para avaliar o cerebelo, porém a medida foi repetida pelo menos três vezes, sendo utilizada para análise a média desses três valores. A mensuração da circunferência abdominal foi obtida mediante corte transversal ao nível da junção da veia umbilical com a veia porta esquerda, sendo obtidas as medidas dos diâmetros ântero-posterior e transverso e então calculada a circunferência em milímetros por meio eletrônico do próprio aparelho.

A medida do DTC de cada feto foi comparada com as medidas esperadas para aquela IG, usando-se a curva de normalidade desenvolvida pelo próprio autor em um grupo de pacientes sem ris$\mathrm{Co}^{15}$. Valores de DTC abaixo do percentil 10 para a IG foram considerados alterados e sugestivos de $\mathrm{RCF}$. A relação DTC/CA foi calculada dividindo o DTC pela CA e multiplicando por 100, conforme estabelecido por Campbell et al. ${ }^{8}$. Conforme recomendado por Meyer et al. ${ }^{10}$, utilizou-se como ponto de corte (cutoff) o percentil 90 da relação DTC/ CA. Baseado em estudo prévio do próprio autor ${ }^{15}$, esse valor foi de 14,6 . Todos os fetos, em qualquer IG, com DTC/CA maior que 14,6 foram considerados como suspeitos para RCF e essas pacientes encaminhadas ao setor de vitalidade fetal para avaliação e conduta individual conforme as condições maternas e fetais específicas ao caso.

Todas as 260 pacientes tiveram seus partos no HUM e seus RN foram examinados por neonatologistas do serviço. Os pesos dos RN foram aferidos usando balança digital e a idade gestacional foi estimada pelo método de Capurro et al. ${ }^{16}$. Os RN com peso inferior ao percentil 10 para sua idade gestacional ${ }^{17}$ foram classificados como portadores de RCF.

Avaliou-se a acurácia do DTC e da relação DTC/CA na detecção de fetos com diagnóstico neonatal de RCF. Foram aferidos a sensibilidade
(S), especificidade (E), valor preditivo positivo (VPP), valor preditivo negativo (VPN) e acurácia dos dois métodos.

Para a análise estatística foi utilizado o programa SPSS - Social Package Statistical Science Versão 8.0 (1997). Foi utilizado o teste $t$ de Student para comparar as diferenças entre os grupos com e sem $\mathrm{RCF}$, sendo fixado nível de significância $\mathrm{p}<0,05$.

\section{Resultados}

Dos 260 fetos estudados, 79 (30,4\%) foram classificados como pequenos para a idade gestacional pelos neonatologistas, recebendo o diagnóstico de RCF. Esses fetos haviam sido avaliados com USG com 33,0 2 2,7 semanas, ao passo que os fetos sem RCF foram avaliados inicialmente na USG com 34,5 $\pm 2,7$ semanas ( $p<0,001)$ em média. A Tabela 1 apresenta a época exata da realização da USG e a distribuição dos fetos classificados como normais ou com $\mathrm{RCF}$ ao nascimento. O intervalo entre a realização da USG e o parto foi de $3,0 \pm 2,2$ semanas para os fetos com restrição de crescimento e de $5,4 \pm 2,8$ semanas para os fetos normais $(\mathrm{p}<0,001)$. A taxa de prematuridade (IG $<37$ semanas) foi de 46,8\% (37 casos) no grupo com $\mathrm{RCF}$ e $15,7 \%$ (41 casos) no grupo sem RCF $(\mathrm{p}<0,001)$. O peso médio dos RN com e sem RCF foi de $2.152 \pm 375$ g e de $2.928 \pm 408 \mathrm{~g}$, respectivamente $(\mathrm{p}<0,001)$.

Tabela 1 - Época da avaliação ultra-sonográfica (USG) dos fetos com e sem restrição de crescimento fetal em 260 gestações de alto risco.

\begin{tabular}{crrrrr}
\hline $\begin{array}{c}\text { Época da USG } \\
\text { (IG em semanas) }\end{array}$ & \multicolumn{2}{c}{ Com RCF* } & \multicolumn{2}{c}{ Sem RCF* } \\
& \multicolumn{1}{c}{$\%$} & \multicolumn{1}{c}{ n } & \multicolumn{1}{c}{$\%$} & Total \\
\hline 28 & 0 & 0,0 & 9 & 5,0 & 9 \\
29 & 0 & 0,0 & 12 & 6,6 & 12 \\
30 & 1 & 1,3 & 16 & 8,8 & 17 \\
31 & 2 & 2,5 & 14 & 7,8 & 16 \\
32 & 5 & 6,3 & 17 & 9,4 & 22 \\
33 & 4 & 5,1 & 21 & 11,6 & 25 \\
34 & 5 & 6,3 & 22 & 12,2 & 27 \\
35 & 9 & 11,4 & 20 & 11,0 & 29 \\
36 & 16 & 20,2 & 20 & 11,0 & 36 \\
37 & 15 & 19,0 & 18 & 10,0 & 33 \\
38 & 10 & 12,7 & 9 & 5,0 & 19 \\
39 & 8 & 10,1 & 3 & 1,6 & 11 \\
40 & 4 & 5,1 & 0 & 0,0 & 4 \\
Total & 79 & 100,0 & 181 & 100,0 & 260 \\
\hline
\end{tabular}

*RCF: Restrição do crescimento fetal (peso abaixo do percentil 10 ao nascer). IG: idade gestacional. 
Em 74 dos 79 fetos com RCF $(93,6 \%)$, o tamanho do cerebelo estava dentro dos limites da normalidade (entre $10^{\circ}$ e $90^{\circ}$ percentis) ${ }^{15}$ na época da realização da USG, ao passo que 5 fetos $(6,4 \%)$ apresentaram DTC abaixo do normal. A Tabela 2 apresenta o desempenho da medida do DTC alterado na detecção do crescimento fetal restrito.

Tabela 2 - Acurácia do diâmetro transverso do cerebelo (DTC) na detecção de fetos com restrição do crescimento.

\begin{tabular}{lccr}
\hline Medida DTC & \multicolumn{3}{c}{ Recém-nascido } \\
& Com RCF & Sem RCF & Total \\
\hline Alterada* $^{*}$ & 5 & 12 & 17 \\
Normal $^{* *}$ & 74 & 169 & 243 \\
Total $^{\text {tal }}$ & 79 & 181 & 260 \\
\hline
\end{tabular}

*Abaixo do percentil 10 para idade gestacional (IG).

${ }^{\star *}$ Entre percentil 10 e 90 para IG.

Sensibilidade $=5 / 79=6,3 \%$; especificidade $=169 / 181=93,4 \%$; valor preditivo positivo $=5 / 17=29,4 \%$; valor preditivo negativo $=169 / 243=69,5 \%$; acurácia $=(5+169) / 260$ $=67,0 \%$.

RCF: restrição do crescimento fetal.

A média da relação DTC/CA para os fetos normais foi de $13,5 \pm 1,0$ e de $15,1 \pm 1,1$ para aqueles com restrição de crescimento $(p<0,001)$. A Figura 1 mostra um gráfico de dispersão das relações DTC/CA dos 260 fetos, evidenciando a tendência dos fetos com RCF (quadrados) se concentrarem acima da linha do percentil 90, ao passo que os fetos normais (estrelas) se situaram predominantemente abaixo do percentil 90.

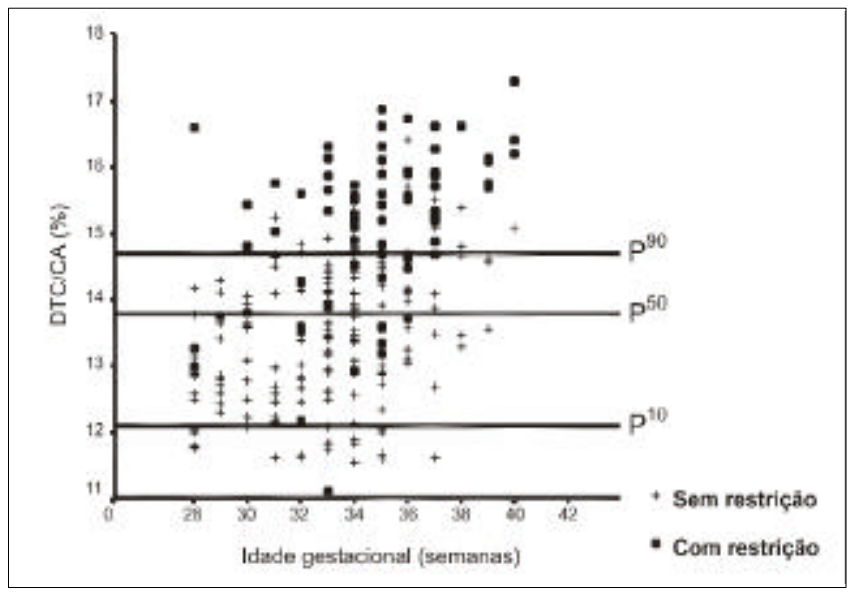

Figura 1 - Gráfico de dispersão com a distribuição dos valores da relação DTC/CA da $28^{\mathrm{a}}$ a $40^{\mathrm{a}}$ semana de gravidez em fetos com e sem restrição de crescimento.

A relação DTC/CA superior ao percentil 90 (ou 14,6) identificou corretamente 59 dos 79 $(74,7 \%)$ RN com restrição de crescimento, teve VPP de $68,6 \%$ e acurácia de $81,9 \%$ neste grupo de pacientes (Tabela 3). Nos 71 casos submetidos a USG nas duas últimas semanas antes do parto, a sen- sibilidade e especificidade do DTC/CA foi de 94,8 e $64,8 \%$, respectivamente. Nos 189 casos avaliados pela USG três ou mais semanas antes do parto, a sensibilidade foi de $57,9 \%$ e a especificidade, de $88,1 \%$.

Tabela 3 - Acurácia da relação diâmetro transverso do cerebelo/circunferência abdominal (DTC/CA) na predição da restrição do crescimento fetal em 260 casos.de risco.

\begin{tabular}{lccr}
\hline Relação DTC/CA & \multicolumn{2}{c}{ Recém-nascido } \\
& Com RCF & Sem RCF & Total \\
\hline Anormal $^{*}$ & 59 & 27 & 86 \\
Normal & 20 & 154 & 174 \\
Total & 79 & 181 & 260 \\
\hline${ }^{*}$ DTC/CA acima do pecentil 90 & & \\
Sensibilidade $=59 / 79=74,7 \%$; especificidade $=154 / 181=85,1 \%$; valor preditivo positivo \\
$=59 / 86=68,6 \%$; valor preditivo negativo $=154 / 174=88,5 \%$; acurácia $=(59+154) / 260$ \\
$=81,9 \%$.
\end{tabular}

\section{Discussão}

Em $16,7 \%$ das 420 pacientes inicialmente elegiveis para o estudo, tivemos dificuldade de medir o cerebelo. Meyer et al. ${ }^{3}$ tiveram dificuldade semelhante em $11 \%$ dos casos, e Haller et al. ${ }^{9}$ em $19 \%$. Embora o cerebelo possa ser visualizado a partir do final do primeiro trimestre de gestação, a obtenção de imagem adequada no terceiro trimestre pode ser prejudicada devido a diversos fatores que dificultam o acesso à fossa posterior do cérebro fetal (obesidade materna, redução do volume de líquido amniótico, insinuação do polo cefálico e apresentação cefálica com o occipício posterior $)^{3,9}$.

A incidência de RCF em nossa população $(30,4 \%)$ foi inferior àquela encontrada por outros autores, que variou de $40,4 \%{ }^{3}$ a $55 \%{ }^{11}$. Tal discrepância pode ser atribuída ao modo de selecionar as pacientes. Enquanto esses autores recrutaram para seus estudos apenas pacientes com relação CC/ CA alterada, incluímos em nosso trabalho todas as pacientes consideradas de risco para restrição de crescimento. Esta diferença de prevalência é dado importante que deve ser lembrado ao compararmos sensibilidade, especificidade e VPP e VPN de um teste. Enquanto os dois primeiros, sensibilidade e especificidade, não são afetados por essas diferenças, os dois últimos (VPP e VPN) aumentam ou diminuem conforme a prevalência de uma doença na população estudada.

Assim como outros trabalhos ${ }^{1-3,8-12}$, o presente estudo evidenciou que a medida do DTC esteve normal na maioria $(93,6 \%)$ dos fetos com RCF. Assim, o DTC isolado demonstrou ser método de baixa sensibilidade $(6,3 \%)$ e alta especificidade $(93.4 \%)$ no rastreamento dessa doença. Esses números são semelhantes aos de Meyer et al. ${ }^{3}$, que 
encontraram sensibilidade e especificidade de 11,3 e $91,0 \%$, respectivamente. Em estudo semelhante, Vinkesteijn et al. ${ }^{18}$ observaram que $26 \%$ dos fetos com RCF tinham diâmetros cerebelares situados abaixo do percentil 10 da curva de normalidade. A constatação de que a maioria dos fetos com RCF tem seu crescimento cerebelar inalterado reforça o valor da medida do DTC para calcular a IG exata de grávidas com fatores de risco para RCF e que se apresentam para USG no $3^{\circ}$ trimestre, sem DUM exata e sem USG prévio.

O mecanismo exato dessa preservação cerebelar ainda não é totalmente conhecido. Estudos realizados há mais de 30 anos em primatas ${ }^{19}$ já demonstravam que, em condições normais, o aporte sanguíneo na região cerebelar é maior que no córtex cerebral e que o cerebelo é um dos últimos órgãos a ser afetado pela diminuição de fluxo sanguíneo. Em fetos asfixiados, embora haja discreto decréscimo de fluxo na região cortical, o fluxo sanguíneo cerebelar permanece inalterado ${ }^{1,3}$.

Neste estudo, a sensibilidade da relação DTC/CA acima do percentil 90 para a detecção da $\mathrm{RCF}$ foi de $74,7 \%$, semelhante aos $71 \%^{11}$ e $73,2 \%{ }^{20}$ apontados por outros autores. Haller et al. ${ }^{9}$ e Meyer et al. ${ }^{3,10}$ foram os autores com os melhores resultados, obtendo sensibilidade de $80 \%{ }^{9}, 83,9 \%{ }^{3} \mathrm{e}$ $87 \%{ }^{10}$. A especificidade observada, $85,1 \%$, foi comparável aos dados da literatura, que variaram de $75 \%{ }^{9}$ até $95,2 \%{ }^{3}$. Quanto aos valores preditivos positivos e negativos observados neste estudo, respectivamente 68,6 e $88,5 \%$, foram inferiores aos citados por Meyer et al. ${ }^{3}(94,5$ e $88,2 \%)$ e por Campbell et al. ${ }^{11}$ (79 e 68\%). Tal diferença, como já comentamos, pode ser atribuída em parte à nossa menor prevalência de RCF (30\%), comparada a esses autores $\left(40,4 \%{ }^{3}\right.$ e $\left.55 \%{ }^{11}\right)$.

Este estudo evidenciou que o diâmetro transverso do cerebelo manteve-se inalterado na maioria dos fetos com RCF, permitindo concluir que a mensuração isolada do DTC não é bom método de rastreamento da RCF. Ao contrário, a relação DTC/ $\mathrm{CA}$ revelou-se eficiente para detectar RCF. Esta relação deveria ser incluída nos exames ultra-sonográficos de rotina e sobretudo naqueles de pacientes de risco para fetos com RCF.

\section{ABSTRACT}

Objective: to evaluate the accuracy of both the transverse diameter of the cerebellum (TDC) and of the transverse diameter/ abdominal circumference (TDC/AC) ratio in the detection of fetal growth restriction (FGR), in high-risk pregnancies.
Method: a prospective cross-sectional study was carried out in 260 patients with gestational age between 28 and 40 weeks. The TDC and AC offetuses were measured through ultrasound and the fetuses with TDC below the 10th percentile or TDC/ $A C$ ratio above the 90 th percentile (>14.6) were classified as FGR suspects. After birth, the accuracy of the TDC and TDC/ $A C$ was evaluated using the neonatal diagnosis of FGR as the gold standard (birth weight $<10$ th percentile).

Results: after birth, 79 newborns (30.4\%) were classified as small for gestational age. The TDC was appropriate in 74 (93.7\%) of these fetuses and small in only $5(6.3 \%)$. The sensitivity (SE), specificity (SP), positive (PPV) and negative $(N P V)$ predictive values and accuracy of the TDC in the prediction of $F G R$ were $6.3,93.4,29.4,69.5$, and $67 \%$, respectively. The TDC/AC >14.6 correctly identified 59 of the 79 growth-restricted fetuses, with 27 false-positives and 20 false-negatives, SE of $74.5 \%$, SP of $85.1 \%$, PPV of $68.6 \%$, NPV of $88.5 \%$ and $81.9 \%$ accuracy.

Conclusion: the TDC is not a good screening parameter for the detection of FGR while the TDC/AC ratio above the 90 th percentile is effective in this detection.

KEYWORDS: Biometry. Cerebellum. Fetal growth restriction.

\section{Referências}

1. Reece EA, Goldstein I, Pilu G, Hobbins JC. Fetal cerebellar growth unaffected by intrauterine growth retardation: a new parameter for prenatal diagnosis. Am J Obstet Gynecol 1987; 157:632-8.

2. Goldstein I, Reece EA. Cerebellar growth in normal and growth-restricted-fetuses of multiple gestation. Am J Obstet Gynecol 1995; 173:1343-8.

3. Meyer WJ, Gauthier D, Ramakrishnan V, Sipos J. Ultrasonographic detection of abnormal fetal growth with the gestational age-independent, transverse cerebellar diameter/abdominal circumference ratio. Am J Obstet Gynecol 1994; 171:1057-63.

4. Manning FA. Intrauterine growth retardation: diagnosis, prognostication, and management based on ultrasound methods. In: Fleischer AC, Manning FA, Jeanty P, Romero R, editors. Sonography in Obstetrics and Gynecology: principles $\&$ practice. $5^{\text {th }}$ ed. London: Prentice-Hall International; 1996. p.51922.

5. Scorza WE, Vintzileos A. First and second trimester sonography: an American perspective. Int $\mathrm{J}$ Fertil Menopausal Stud 1996; 41;288-92.

6. McLeary RD, Kuhns LR, Barr M Jr. Ultrasonography of the fetal cerebellum. Radiology 1984; 151:439-42.

7. Goldstein I, Reece EA, Pilu G, Bovicelli L, Hobbins JC. Cerebellar measurements with ultrasonography in the evaluation of fetal growth and development. Am J Obstet Gynecol 1987; 156:1065-9. 
8. Campbell WA, Nardi D, Vintzileos AM, Rodis JF, Turner GW, Egan JF. Transverse cerebellar diameter/abdominal circumference ratio throughout pregnancy: a gestational age-independent method to assess fetal growth. Obstet Gynecol 1991; 77:893-6.

9. Haller H, Petrovic O, Rukavina B. Fetal transverse cerebellar diameter/abdominal circumference ratio in assessing fetal size. Int J Gynaecol Obstet 1995; 50:159-63.

10.Meyer WJ, Gauthier DW, Goldenberg B, Santolaya J, Sipos J, Cattledge F. The fetal transverse cerebellar diameter/abdominal circumference ratio: a gestational age-independent method of assessing fetal size. J Ultrasound Med 1993; 12:379-82.

11.Campbell WA, Vintzileos AM, Rodis JF, Turner GW, Egan JF, Nardi DA. Use of the transverse cerebellar diameter/abdominal circumference ratio in pregnancies at risk for intrauterine growth retardation. J Clin Ultrasound 1994; 22:497-502.

12.Lee W, Barton S, Comstock $\mathrm{CH}$, Bajorek S, Batton D, Kirk JS. Transverse cerebellar diameter: a useful predictor of gestational age for fetuses with asymmetric growth retardation. Am J Obstet Gynecol 1991; 165:1044-50.

13.Hill LM, Guzick D, Rivello D, Hixson J, Peterson C. The transverse cerebellar diameter cannot be used to assess gestational age in the small for gestational age fetus. Obstet Gynecol 1990; 75:329-33.
14.Cabbad M, Kofinas A, Simon N, King K, Lyttle E. Fetal weight-cerebellar diameter discordance as an indicator of asymmetrical fetal growth impairment. J Reprod Med 1992; 37:794-8.

15.Nery L, Moron AF, Kulay Junior L. Avaliação ultrasonográfica do crescimento fetal com o uso do diâmetro transverso do cerebelo. Rev Bras Ginecol Obstet 2000; 22:281-6.

16. Capurro H, Konichezky S, Fonseca D, Caldeyro-Barcia R. A simplified method for diagnosis of gestational age in the newborn infant. J Pediatr 1978; 93:120-2.

17. Battaglia FC, Lubchenco LO. A practical classification of newborn infants by weight and gestational age. J Pediatr 1967; 71:159-63.

18.Vinkesteijn AS, Mulder PG, Wladimiroff JW. Fetal transverse cerebellar diameter measurements in normal and reduced fetal growth. Ultrasound Obstet Gynecol 2000; 15:47-51.

19.Behrman RE, Lees MW, Peterson EN, De Lanoy CW, Seeds AE. Distribution of the circulation in the normal and asphyxiated fetal primate. Am J Obstet Gynecol 1970; 108:956-69.

20.Tongsong T, Wanapirak C, Thongpadungroj T. Sonographic diagnosis of intrauterine growth restriction (IUGR) by fetal transverse cerebellar diameter (TCD)/abdominal circumference (AC) ratio. Int J Gynaecol Obstet 1999; 66:1-5.

Recebido em: 14/10/2002 Aceito com modificações em: 29/3/2004 